AN. MED INTERNA (Madrid) Vol. 18, N. $^{\circ} 4$, pp. 181-186, 2001

\title{
Fiebre de origen desconocido en pacientes VIH positivos
}

\author{
R. BARBA, J. GÓMEZ-RODRIGO, J. MARCO, P. RONDÓN, G. EROLES, \\ M. LÓPEZ-VARAS, R. TORRES \\ Departamento de Medicina Interna y Enfermedades Infecciosas. Hospital Severo Ochoa. \\ Leganés. Madrid
}

\author{
FEVER ON UNKNOWN ORIGIN IN PATIENTS WITH HIV
}

\section{RESUMEN}

Objetivos: Describir la presentación clínica y la utilidad de los de tests diagnósticos habitualmente recomendados en el estudio de la fiebre de origen desconocido (FOD) en los pacientes VIH positivos.

Pacientes y métodos: Incluimos en el estudio a los 54 pacientes con infección por el VIH que ingresaron en nuestro Hospital por FOD durante un periodo de 23 meses. La FOD fue definida de acuerdo con los criterios modificados de Petersdorf's.

Resultados: La causa de la fiebre se identificó en 48 casos (89\%). La tuberculosis, la micobacteriosis atípica y la leishmaniasis pueden explicar el $68 \%$ de los casos. El aspirado de médula ósea, la punción aspiración o la biopsia de los ganglios linfáticos y los cultivos para micobacterias fueron las pruebas diagnósticas más rentables.

Conclusiones: La infección por micobacterias debe ser el primer diagnóstico de sospecha en los pacientes VIH positivos con FOD. Es posible precedir el diagnóstico de tuberculosis con una alta precisión $(90,5 \%)$ con un modelo de regresión logística basado en datos clínicos y analíticos fácilmente obtenibles.

\begin{abstract}
Objectives: To describe the clinical presentation of FUO in patients infected with HIV and to asses the diagnostic usefulness in this popula tion of several test usually recommended in the study of FUO.

Patients and methods: We studied the clinical charts of all patients with HIV that required admission to our Hospital during a 23-month period. FUO was defined according to Petersdorf's modified criteria. Fifty-four patients fulfilled study criteria during the entry period.

Results: A cause of fever was identified for 48 patients (89\%). Tuber culosis, disseminated atypical mycobacteriosis and Leishmaniasis can explain 68\% of them. Examination of bone marrow aspirates, lymph node aspirates and biopsy, and culture of clinical specimens for myco bacteria were the procedures with the highest diagnostic yield.

Conclusions: Mycobacterial infection should be considered as a first-line diagnosis in HIV-patients with FUO. It is possible to predict the diagnosis of tuberculosis infection with a high level of confidence (90.5\%) through a logistic regression model based on easily obtainable parameters.
\end{abstract}

PALABRAS CLAVE: Fiebre de origen desconocido (FOD). HIV. Sida. Micobacterias.

KEY WORDS: Fever of unknown origin. HIV. AIDS. Mycobacteria.

Barba R, Gómez-Rodrigo J, Marco J, Rondon P, Eroles G, López-Varas M, Torre R. Fiebre de origen desconocido en pacientes HIV positivos. An Med Interna (Madrid) 2001; 18: 181-186.

\section{INTRODUCCIÓN}

La fiebre es uno de los motivos más frecuentes de ingreso hospitalario en los pacientes con infección por VIH (1-2), representando la Fiebre de Origen Desconocido (FOD) un 5$21 \%$ de todos los ingresos hospitalarios en pacientes con SIDA según algunas series (3-7). El uso de métodos diagnósticos agresivos en la evaluación de estos pacientes y la introducción de tratamientos empíricos precoces, son temas que aún permanecen debatidos. La posibilidad de predecir algunos de los diagnósticos más frecuentes de FOD mediante modelos matemáticos podría tener interés a la hora de iniciar trata- mientos empíricos, que suelen ser tóxicos, en pacientes, por otro lado habitualmente polimedicados.

Por otro lado parece interesante identificar aquellos métodos diagnósticos que sean más útiles a la hora de llegar al diagnóstico de FOD, o determinar en que situaciones parece más justificado el uso de técnicas agresivas o caras, por su mayor rentabilidad.

Las preguntas a estas cuestiones sólo pueden responderse mediante la realización de estudios prospectivos que tengan un seguimiento lo suficientemente amplio como para permitir conocer el diagnóstico y la evolución del mayor número de pacientes. Por ello nos propusimos realizar un estudio pros-

Trabajo aceptado: 19 de Septiembre de 2000

Correspondencia: J. Gómez-Rodrigo. Hospital Severo Oochoa. Avda. Orellana s/n. 28911 Leganés (Madrid). 
pectivo en pacientes VIH positivos con FOD, cuyos principales objetivos eran describir las características clínicas, las pruebas diagnósticas de mayor rentabilidad en su estudio, los factores predictores de mortalidad y un modelo matemático capaz de identificar los factores predictores de infección tuberculosa.

\section{MATERIAL Y MÉTODOS}

Se diseñó un estudio prospectivo, observacional, en el que se incluyeron los pacientes infectados por el VIH que ingresaron en el hospital Severo Ochoa durante los meses de febrero de 1995 y diciembre de 1996 que tenían fiebre de origen desconocido (FOD). Se trata de un Hospital de segundo nivel, que dispone de 425 camas y atiende a una población de 400.000 personas. Los criterios de inclusión fueron los establecidos Petersdorf (8) para los pacientes con infección VIH: presencia de fiebre superior a $38,3^{\circ} \mathrm{C}$ medida en varias ocasiones a lo largo de un periodo de más de 4 semanas en pacientes ambulatorios, o de más de 3 días en los pacientes hospitalizados. El diagnostico se establecía cuando un estudio adecuado de 3 días de duración (incluidos al menos dos días de incubación de los cultivos) no revelara la causa. Este estudio debía incluir hemocultivos, cultivos de orina, radiografía de tórax, hemograma, bioquímica de sangre y orina y exploración física habitual.

En los casos que cumplían los criterios se cumplimentó un protocolo clínico que recogía datos demográficos (sexo, edad), modo de contagio (uso de drogas por vía parenteral, relaciones homosexuales o heterosexuales, transfusiones de sangre o hemoderivados, desconocido), diagnóstico de sida según los criterios de los CDC (9), enfermedades diagnósticas de sida previas, medicaciones antirretrovirales utilizadas, último recuento de CD4 (en los 6 meses previos al ingreso). Asimismo se incluyeron datos referentes al episodio actual que incluía determinaciones analíticas (recuentos de leucocitos, hematocrito, plaquetas, glucosa, creatinina, transaminasas (GOT, GPT), velocidad de sedimentación glomerular, datos de la exploración clínica (presencia de soplos, adenomegalias, organomegalias, lesiones cutáneas) y de síntomas asociados (tos, disnea, expectoración, pérdida de peso, astenia, anorexia, ictericia, cefalea, dolor abdominal).

Los pacientes fueron sometidos a pruebas diagnósticas a criterio de sus médicos en función de los síntomas clínicos y los resultados que se iban obteniendo. Cada una de las pruebas solicitadas y sus resultados fueron registrados en el protocolo. Las pruebas solicitadas incluían: ecografía o TAC abdominal; cultivo de esputo, líquido cefalorraquídeo, sangre y orina para micobacterias; biopsia de piel; biopsia o punción aspiración con aguja fina de adenopatías procesada para estudio anatomopatológico y cultivo de micobacterias; punción aspiración de médula ósea procesada para estudios anatomopatológico y cultivo de micobacterias y Leishmania; TAC craneal; sexología de LUES, CMV, Leishmania, Fiebre Q y Brúcela; fondo de ojo, punción lumbar.

Se consideraba enfermedad diagnóstica aquella que tuvieran confirmación microbiológica, histológica o seroconversión. Se definió tiempo hasta el diagnóstico como el número de días que se precisaba para obtener el diagnóstico definitivo desde que el paciente era ingresado o diagnosticado definitivamente de FOD (excluyendo los 3 primeros días necesarios para establecer los criterios diagnósticos). El inicio de tratamiento empírico (considerándose como tal al que se iniciaba antes de obtenerse los resultados confirmatorios) se dejó a criterio del médico responsable de cada paciente. No se consideró diagnóstica una respuesta clínica (desaparición de la fiebre) tras iniciar tratamiento empírico.

Todos los pacientes fueron seguidos durante intervalos regulares hasta su muerte o hasta cumplir dos años tras el ingreso. Se consideró muerte relacionada aquella ocurrida durante el seguimiento que no pudiera ser justificada por otra circunstancia (toxicidad farmacológica, presencia inequívoca de otra enfermedad una vez resuelto el proceso agudo, accidente de tráfico etc.).

Se realizó un análisis descriptivo de las variables demográficas, clínicas y los datos analíticos. Se compararon los pacientes con y sin diagnóstico al final del seguimiento, y los pacientes que habían fallecido frente a los que no lo hicieron, utilizando el test de chicuadrado para variables categóricas con corrección de Yates y el test exacto de Fisher para las variables dicotómicas cuando el valor esperado de una celda era menor de 5. Las variables cuantitativas se compararon mediante el test de la T. Aquellas variables que tuvieron una significación $<0,10$ en el análisis bivariante o si tenían relevancia clínica se introdujeron en modelo multivariante de regresión logística con el fin de identificar los factores que se correlacionaran de forma independiente con la mortalidad. Asimismo se introdujeron las variables que se correlacionaban con el diagnóstico de tuberculosis en otro modelo, con el fin de determinar los factores predictores de tuberculosis. La estimación de los modelos se hizo según el método de estimación máxima de verosimilitud hacia atrás por pasos.

Se realizó un análisis de supervivencia de Kaplan-Meier para determinar la proporción de pacientes vivos en los grupos con y sin diagnóstico, así como en el grupo con diagnóstico de tuberculosis frente a los que tenían otros diagnósticos. Como el método de Kaplan-Meier tiene limitaciones al no poder considerar el efecto de otros factores, también se realizó un análisis de Cox con intención de determinar el riesgo de mortalidad asociado con la ausencia de diagnóstico, o con el diagnóstico de tuberculosis. Realizamos un modelo de Cox, en el cual la muerte fue considerada como una variable dependiente del tiempo y se ajustó por factores demográficos y otros posibles factores relevantes.

Todos los análisis estadísticos se realizaron con el SPSS para Windows versión 7,5 (10).

\section{RESULTADOS}

Durante los 23 meses de reclutamiento, de un total de 377 pacientes con infección VIH ingresados en nuestro centro, 54 pacientes fueron incluidos en el estudio (14\%). La estancia media de estos pacientes fue de 27 días (4-68) comparados con una estancia media de 13 días del grupo total de pacientes VIH ingresados: eso implica una estancia acumulada de 1378 días de un total de 4.895 , lo que supone que el $14 \%$ de los pacientes consumieron el $28 \%$ del tiempo. La edad media de los pacientes fue de $33,7 \pm 5,2$ años y un $26 \%$ de los pacientes eran mujeres. El 68,5\% (37 casos) habían adquirido la infección por uso de drogas por vía parenteral, en 3 casos $(5,6 \%)$ por relaciones homosexuales, $10(18,5 \%)$ por contacto hetero- 
sexual y en 4 casos $(7,4 \%)$ se desconocía el modo de adquisición de la infección. No hay diferencias entre este subgrupo de pacientes y nuestra población habitual de pacientes.

La media de CD4 fue de 113 células/L (rango 1-660); 34 pacientes $(63 \%)$ eran clasificados en el grupo $\mathrm{C}$ de los CDC de1993 (9), 18 (33,3\%) en el grupo B y $2(3,7 \%)$ en grupo A.

Se llegó al diagnóstico definitivo en 48 pacientes $(89 \%)$ (Tabla I). El tiempo necesario para llegar al diagnóstico fue de 24,5 días (7-65) con una duración media de la fiebre antes del ingreso hospitalario de 57,6 $\pm 35,6$ días (21-180), lo cual significa una media de 85 días (42-188) de duración media del episodio febril. Tres enfermedades: la infección por micobacterias tuberculosas, la infección por micobacterias atípicas y la leishmaniasis visceral explican el $68 \%$ de los episodios de FOD en nuestra serie (37 casos). En 6 de nuestros pacientes (11\%) existía otra enfermedad concomitante potencialmente responsable de la fiebre: dos candidiasis esofágicas, dos retinitis por CMV y dos toxoplasmosis cerebrales. En todos los casos el curso clínico de la enfermedad descartó estas otras entidades como responsables de la fiebre.

\begin{tabular}{lr}
\multicolumn{2}{c}{ TABLA I } \\
\multicolumn{1}{c}{ CAUSAS DE FO D EN PACIENTES CO N INFECCIÓ N VIH* } \\
\hline Causa & No $(\%)$ de pacientes \\
\hline Tuberculosis & $22(41 \%)$ \\
Infección por micobacterias atípicas & $12(22 \%)$ \\
Leishmaniasis visceral & $5(9 \%)$ \\
Criptococcosis & $2(3,7 \%)$ \\
Linfoma No-Hodgkin & $2(3,7 \%)$ \\
Infección diseminada por CM V & $3(5,6 \%)$ \\
Toxoplasmosis & $1(2 \%)$ \\
Prostatitis Chronica & $1(2 \%)$ \\
Sin identificar & $6(11 \%)$ \\
\hline
\end{tabular}

* En $11 \%$ de los pacientes más de una causa fue identificada. La table incluye la enfermedad que más probablemente fue responsable del cuadro de FOD.

El síntoma focal más frecuente fue la tos (35 casos, $64,8 \%$ ), aunque la mayoría de los pacientes presentaban síntomas o signos de más de un órgano o sistema, y en casi todos los casos (89\%) los síntomas no coexistían exactamente en el tiempo con la fiebre, sino que la precedían o habían aparecido un tiempo después que esta.

Con relación a las investigaciones de rutina realizadas, la media de la hemoglobina, leucocitos y plaquetas fue de $10,2 \pm 2,3 \mathrm{~g} / \mathrm{dL}$ (6-15), 3.810 $\pm 2.793 / \mathrm{ml}$ (380-16.000), 163.000 $\pm 78.000 / \mathrm{mm}^{3}(9.000-326.000)$ respectivamente. En 20 casos (37\%) los pacientes presentaban alteraciones de la función hepática (considerada como 3 veces los valores normales) con predominio de los pacientes con colostasis (35\%) sobre citolisis $(11 \%)$.

En el estudio de los pacientes se realizaron numerosas pruebas diagnósticas, tanto invasivas como no invasivas. La media de pruebas por paciente (excluyendo análisis rutinarios) fue de 10 (5-30) (Tablas II y III). El aspirado de médula ósea, la biopsia y cultivo de diferentes muestras fueron los procedimientos que mostraron mayor rentabilidad, sobre todo para identificar infecciones por micobacterias. En el $21 \%$ de los casos el diagnóstico fue realizado por aspirado de médula y en el $19 \%$ por cultivo de esputo. Los cultivos de sangre para micobacterias resultaron positivos y diagnósticos en 8 casos de un total de $45(17 \%)$.

TABLA II

PRINCIPALES PRO CEDIM IENTOS DIAGNÓ STICOS UTILIZADOS PARA IDENTIFICAR LA CAUSA DE FOD EN PACIENTES VIH+

\begin{tabular}{lccr}
\hline Procedimiento & № realizados & № positivos (\%) & № diagnosticos \\
\hline $\begin{array}{l}\text { Aspirado de } \\
\quad \text { médula ósea }\end{array}$ & 28 & $12(43 \%)$ & $10(18,5 \%)$ \\
$\begin{array}{l}\text { Biopsia de ganglio } \\
\text { O tras biopsias }\end{array}$ & 11 & $6(54 \%)$ & $5(9 \%)$ \\
Cultivos micobacterias & 4 & $4(100 \%)$ & $4(7 \%)$ \\
Esputo & 46 & $18(39 \%)$ & $9(16 \%)$ \\
Sangre & 46 & $8(17 \%)$ & $4(7 \%)$ \\
LCR† & 23 & $2(9 \%)$ & $2(3,7 \%)$ \\
Orina & 17 & $3(18 \%)$ & \\
Broncoaspirado & 8 & $2(25 \%)$ & $2(3,7 \%)$ \\
Cultivo de orina & 37 & $3(8 \%)$ & $1(2 \%)$ \\
Serolgía CM V & 21 & $4(19 \%)$ & $1 *(2 \%)$ \\
\hline
\end{tabular}

† LCR: Líquido cefalorraquídeo. *El diagnóstico se hizo por seroconversión.

TABLA III OTRO S PRO CEDIM IENTOS DIAGNÓ STICOS

\begin{tabular}{lcr}
\hline & No Realizados & № Patológicos (\%) \\
\hline Radiografía de tórax & 54 & $14(26)$ \\
Hemocultivos & 53 & $2(4)$ \\
Sexología (Fiebre Q, brucella, ś́filis) & 52 & 0 \\
Hemocultivos de larga incubación & 45 & 0 \\
Fondo de ojo & 35 & $8(23)$ \\
Coprocultivos & 33 & $2(6)$ \\
Identificación de parásitos en heces & 33 & $2(6)$ \\
Ecografía abdominal & 28 & $21(75)$ \\
Punción Lumbar & 23 & $8(35)$ \\
TC cranial & 20 & $6(30)$ \\
Ecocardiografía & 14 & 0 \\
TC torácico & 11 & $8(73)$ \\
Broncoscopia & 8 & $4(50)$ \\
\hline
\end{tabular}

Se hicieron sistemáticamente serologías de sífilis, Leishmania, Fiebre Q, Brucela y CMV, aunque su impacto en el diagnóstico definitivo fue prácticamente nulo. Solamente en un caso una seroconversión en la sexología de CMV sirvió como diagnóstico (el paciente desarrollo una retinitis por CMV en el curso del tratamiento con ganciclovir; el tratamiento con foscarnet mejoró los síntomas generales y ocula- 
res). La radiografía de tórax fue normal en 40 casos (74\%). Las alteraciones presentes se categorizaron en cuatro grupos: patrones alveolares, intersticiales, ensanchamiento mediastínico y afectación pleural con una incidencia del 11, 7 y $3 \%$ y $3 \%$ respectivamente. Los hallazgos radiológicos no se correlacionaron con el diagnóstico final. Se realizó punción lumbar en $23(43,4 \%)$; en 8 de ellos hubo alteraciones que consistieron fundamentalmente en elevaciones del nivel de las proteínas. En cuatro de esos casos el diagnóstico final fue de una enfermedad que afectaba al sistema nervioso central (1 criptococosis, y 3 meningitis tuberculosas). El examen del fondo de ojo fue normal en el $68 \%$ de los casos y no contribuyó en ningún caso al diagnóstico final de FOD en nuestra serie.

Se inició tratamiento empírico en 31 pacientes $(57,4 \%)$, y $12(22 \%)$ casos se hizo más de un tratamiento empírico durante el curso de la enfermedad. En el $32 \%$ de los casos el diagnóstico de sospecha coincidió con el diagnóstico final, siendo la tasa de acierto mas alta en el caso de sospecha de tuberculosis $(50 \%$ de los casos que se puso tratamiento antituberculoso se acertó). En 22 casos $(42,6 \%)$ no se inició ningún tratamiento empírico: no hubo diferencias en cuanto a la mortalidad entre los dos subgrupos de pacientes.

No se llegó al diagnóstico final en 6 pacientes, cuatro de los cuales fallecieron durante el ingreso. En un sólo caso la fiebre se autolimitó. El número de prodecimientos diagnósticos realizados a estos pacientes fue algo superior que el realizado al grupo total, aunque no alcanzó significación estadística (12,1 versus $10,2, p=0,248)$. Las muertes en estos pacientes fueron debidas a distres respiratorio. La mortalidad intrahospitalaria fue claramente superior entre los pacientes sin diagnóstico que en los que fueron diagnosticados (66\% versus $8,5 \%$; $<<0,0001)$, así como la mortalidad al cabo de 2 años de seguimiento (100\% versus $61 \% ; \mathrm{p}=0,08$ )

Durante el periodo de estudio 34 pacientes fallecieron $(63 \%$ del total), la mortalidad intrahospitalaria durante el periodo de estudio fue del $15 \%$ y el $40,7 \%$ fallecieron en los 6 meses siguientes al diagnóstico. El riesgo de morir fue superior en los pacientes con diagnóstico diferente al de tuberculosis, incluso una vez que se corregía por posibles confusores, como la edad, las cifras de CD4, o el tiempo de evolución de la enfermedad.

Veintidós de los 54 pacientes fueron diagnosticados de tuberculosis. Con el fin de identificar las variables que podían ayudar a predecir el diagnóstico de tuberculosis en los pacientes con FOD se realizó un análisis multivarainte mediante un modelo de regresión logística. Se incluyeron en el análisis las variables cuya significación en el análisis bivariante fuera $<0,1$ y aquellas que tenían relevancia clínica; así fueron incluidas: PPD; total de días con fiebre previos al ingreso; ausencia de dolor abdominal y disnea como datos clínicos previos; presencia de adenopatías y crepitantes en la exploración física, kilos perdidos durante el episodio febril; cifra de CD4, leucocitos, plaquetas, hemoglobina y triglicéridos. Los datos relacionados con el diagnóstico de tuberculosis fueron un PPD positivo y las cifras de CD4, triglicéridos y plaquetas (los pacientes con diagnóstico final de tuberculosis tenían cifras de plaquetas y de CD4 por encima de $140.000 / \mathrm{ml}$ y $200 / \mathrm{ml}$ respectivamente, triglicéridos por debajo de $130 \mathrm{mg} / \mathrm{dl}$ ). Con este modelo el 90,5\% de los pacientes quedaba bien clasificado.

La proporción de pacientes vivos después de un seguimiento medio de $11,8 \pm 10,9$ meses fue del $5 \%$ ( 1 de 18 ) en el grupo de pacientes a los que se diagnosticó de tuberculosis frente a un 51\% (17 de 33) de los pacientes que tuvieron otros diagnósticos. Las curvas de supervivencia en los dos grupos fueron significativamente diferentes (log-rank test; $13.4 ; \mathrm{p}=$ 0,0003). El $100 \%$ de los pacientes sin diagnóstico había fallecido tras un año de seguimiento frente a un $40 \%$ de los pacientes que habían sido diagnósticados (log-rank test: 22,6; $\mathrm{p}<0,0001$ ) (Fig. 1). En el modelo de Cox se introdujeron como variables presuntamente predictoras de mortalidad: la edad, el sexo, el factor de riesgo de infección VIH, la cifra de CD4, la hemoglobina y los niveles de triglicéridos, los días de fiebre, la pérdida de peso, el que se hubiera obtenido un diagnóstico durante el episodio de FOD. Los predictores independientes de mortalidad fueron: la ausencia de diagnóstico (RR: 9,7; 95\% CI: 2,9-32,1; $\mathrm{p}=0,0002)$, la cifra de triglicéridos (RR $1,4,95 \%$ CI: $1,3-1,7 ; \mathrm{p}=0,009)$ y la hemoglobina (RR $0,7,95 \%$ CI $0,6-0,8, \mathrm{p}=0,002)$.

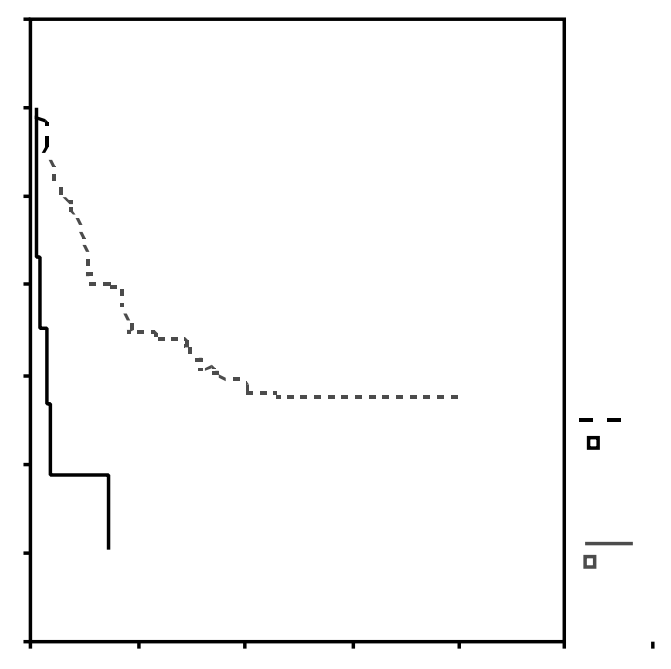

Fig. 1. Supervivencia acumulada en los pacientes con y sin diagnóstico.

\section{DISCUSIÓN}

La fiebre de origen desconocido es un problema frecuente entre los pacientes con infección por el virus de la inmunodeficiencia humana. Aunque somos capaces de diagnosticar a la mayoría de los pacientes que presentan este cuadro, un $86 \%$ en nuestra serie, cifra que se asemeja mucho a lo publicado en otros trabajos similares (3-7), esto supone un importante gasto sanitario, debido a las numerosas pruebas que es necesario realizar y a la larga estancia media que condicionan estos procesos. La infección tuberculosa es la causa más frecuente de FOD en nuestros pacientes (22 casos, $46 \%$ de los diagnósti$\cos )$, dato que ya había sido demostrado en otras series realizadas en nuestro país (5-7). Sin embargo los 12 casos (22\% de 
los pacientes estudiados) de infección por micobacterias atípicas suponen una incidencia claramente superior a las series realizadas tanto en nuestro país (5-7) como fuera de él (3-4), en los que se ha publicado una incidencia que oscila entre el 10 y el $15,5 \%$. Esto podría explicarse por que nuestra serie es prospectiva y se buscaron específicamente las micobacteriosis atípicas.

Como se ha observado en la mayor parte de las series publicadas hasta ahora (3-7), el diagnóstico más frecuente en los pacientes VIH positivos con FOD en nuestro medio es el de tuberculosis. Utilizando un modelo predictivo podemos diagnosticar de tuberculosis a los pacientes con FOD con una fiabilidad del $90 \%$. Los pacientes VIH positivos con FOD, sin inmunosupresión severa ni trombopenia, con cifras de triglicéridos bajas y un PPD positivo tienen una alta probabilidad de tuberculosis, por lo que nosotros consideramos que este subgrupo de pacientes podría ser subsidiario de recibir tratamiento tuberculostático empírico, mientras se esperan los resultados de los cultivos. El resto de los pacientes, con datos que indican una inmunosupresión más severa y desnutrición/afectación general más importante (medida como aumento de triglicéridos) (11), existen otras posibilidades diagnósticas, por lo tanto el tratamiento empírico con tuberculostáticos no estaría, en principio, justificado.

Existen dos factores sorprendentes en nuestra serie: en primer lugar la ausencia de casos de FOD relacionados con uso de fármacos, como han descrito otros autores $(4,5,7)$. Esto podría explicarse porque en nuestro centro se retiraban sistemáticamente todos los fármacos en la primera fase del estudio de la FOD. En segundo lugar puede resultar sorprendente la exclusión de la biopsia hepática como parte del estudio diagnóstico en nuestra serie, sobre todo si tenemos en cuenta la alta proporción de nuestros pacientes que tenían alteraciones en la bioquímica y los datos publicados en otras series $(6,12,13)$. La explicación a esto puede encontrarse en las dificultades que existen en nuestro centro para realizar esta prueba. Dado que todos los pacientes que fallecieron sin diagnóstico lo hicieron por causas respiratorias, parece razonable asumir que dicha técnica no hubiera ampliado el número de casos diagnosticados. Además mientras algunos autores han demostrado que la biopsia hepática una de las técnicas más rentables en el estudio de FOD $(6,13)$, otras series demuestran que su utilidad es limitada dado que se puede obtener el diagnóstico mediante otras pruebas menos invasivas (14). Posiblemente la utilidad de la prueba esté en parte condicionada por la accesibilidad y la experiencia que exista en cada centro.

Es especialmente importante determinar la utilidad de los test que usamos en la evaluación de los pacientes con FOD, para poder establecer unas prioridades a la hora de solicitar las pruebas diagnósticas (15). El papel del aspirado de médula ósea en el diagnóstico de FOD en pacientes VIH ha sido debatido con frecuencia (16-20). Mientras algunos autores han publicado rentabilidades muy bajas $(16,17)$ otros lo consideran muy útil y lo recomiendan cuando se precisa un rápido diagnóstico o si las pruebas rutinarias no han permitido llegar al diagnóstico etiológico $(5,18-20)$. En nuestra serie el aspirado de médula ósea resulta un método diagnóstico bastante útil, permitiendo el diagnóstico en del $21 \%$ de los casos. Cuando los pacientes fueron diagnosticados de
Leishmaniasis fue el único test que resultó diagnóstico, ya que la sexología tiene una sensibilidad muy baja (el 60\% de los pacientes con leishmaniasis tenían una sexología negativa). También resulta especialmente útil para el diagnóstico de Micobacteriosis atípicas. Teniendo en cuenta que en nuestro medio ambos diagnósticos son frecuentes creemos que el aspirado de medula es una técnica que debe utilizarse de forma rutinaria y precoz en la evaluación de los pacientes con FOD

Existe una relación entre la duración de la fiebre y el diagnóstico final, siendo menor la duración en los pacientes que tiene un diagnostico final de tuberculosis (77 días) y más larga en los pacientes con micobacteriosis atípica (106 días; $\mathrm{p}=0,03$ ). Posiblemente esto pueda explicarse por las dificultades que existen para el cultivo de las micobacterias atípicas, y porque en muchos casos la clínica es más larvada y en pacientes con enfermedad más avanzada lo que les puede hacer acudir más tarde al médico. No hemos encontrado relación entre la práctica de riesgo, el uso previo de antirretrovirales o de otros fármacos y el diagnostico final. La mayoría de nuestros pacientes habían recibido mono o biterapia previamente o durante el proceso febril, dado que cuando se inició el estudio aún no se había generalizado en nuestro medio el uso de inhibidores de proteasa ni de terapias combinadas. Este aspecto puede impedir que los resultados epidemiológicos sean extensibles a la época en la que la triple terapia es de uso común (10).

La mortalidad en la mayoría de las series es alta (4-7), cosa que corroboramos en nuestro estudio. Los pacientes sin diagnóstico final tienen mucho más riesgo de fallecer tanto durante el ingreso como durante los dos años que siguen al episodio que los pacientes diagnosticados, lo que refuerza la hipótesis de la importancia del diagnóstico en estos pacientes. Los pacientes con diagnóstico de tuberculosis tienen un mejor pronóstico que los que son diagnosticados de otras enfermedades. Dado que nuestra serie está hecha en pacientes que no recibían tratamiento antirretroviral intensivo su mortalidad parece estar en relación con la evolución de su enfermedad, pero la inmunosupresión no explica por si misma la mortalidad de estos pacientes. Son otros datos los que se correlacionan de forma mas directa con la mortalidad en nuestra serie. La ausencia de diagnóstico aumenta nueve veces el riesgo de fallecer. También lo hacen la desnutrición del paciente, medida como aumento de triglicéridos, como ya ha sido publicado previamente (11), o la anemia.

La mayoría de los pacientes de nuestra serie con FOD tienen una enfermedad que puede diagnosticarse y tratarse (15), por lo que están justificados estudios agresivos para intentar llegar al diagnóstico. En un subgrupo de pacientes, con menor inmunosupresión y con PPD positivo las posibilidades de que el diagnóstico final sea de tuberculosis son altas, por lo que creemos justificado un tratamiento empírico en estos casos. La generalización del uso de tratamiento antirretroviral ha podido modificar la incidencia de la FOD y puede que también el espectro de enfermedades que aparecen relacionadas con este cuadro. Sin embargo en nuestro medio posiblemente hoy también sigan siendo las micobacteriosis y la leishmania las enfermedades más frecuentes, por lo que debemos protocolizar el estudio incluyendo pruebas diagnósticas que nos permitan llegar al diagnóstico de estos procesos. 


\section{Bibliografía}

1. Sullivan M, Feinberg J, Barlett J;G. Fever in patients with HIV infection. Infect Dis Clin North Am 1996; 10: 149-165.

2. Barat LM, Gunn JE; Steger KA, Perkins CJ, Craven DE. Causes of fever in patients infected with human immunodeficiency virus who were admitted to Boston City Hospital. Clin Infect Dis 1996; 23: 320-328.

3. Genne D, Chave JP, Glauser MP. Fever of unknown origin in a cohort of HIV-positive patients. Schweiz Med Wochenschr 1992; 122: 1797-1802.

4. Bissuel F, Leport C, Perronne C, Longuet P, Vilde JL. Fever of unknown origin in HIV-infected patients: a critical analysis of a retrospective series of 57 cases. J Intern Med 1994; 236: 529-535.

5. Miralles P, Moreno S, Perz-Tascon M, Cosin J, Diaz MD, Bouza E. Fever of uncertain origin in patients infected with the human immunodeficiency virus. Clin Infect Dis 1995; 20: 872-875.

6. Lozano F, Torres-Cisneros J, Bascuñana A, Polo J, Viciana P et al. Prospective evaluation of fever of unknown origin in patients infected with the human immunodeficiency virus. Grupo Andaluz para Estudio de las Enfermedades Infecciosas. Eur J Clin Microbiol Infect Dis 1996; 15: 705-711.

7. Carbonell Biot J, Ena Muñoz J, Pasquau Liaño F, Badia Ferrando P. Ortiz de Salazar Martín A, Vilar Zanón A. Fever of uknown origin in patients infected with human immunodeficiency virus. Rev Clin Esp 1996; 196: 4-8.

8. Durak DT, Street AC. Fever of unknown origin, reexamined and redefined. Curr Clin Top Infect Dis 1991; 11: 35-51.

9. CDC. Revised classification system for HIV infection and expanded surveillance case definition for AIDS among adolescents and adults MMWR 1992; 41: 17.

10. SPSS. Stadistical Package for Social Sciences (computer program). Windows version, release 7,5. Chicago SPSS 1997.

11. Estado nutricional de pacientes con infección por VIH. Evolución espontánea durante un ingreso hospitalario. D. del Olmo, V. Alcazar, R. Barba, C. Fernandez, M. A. Koning, C. Vazquez. Nutrición Hospitalaria 1997; 206-209.

12. Cavicchi M, Pialoux G, Carnot F, Offredo C, Romana D, Deslandes P, Dupont B, Berthelot P, Pol S. Value of liver biopsy for the rapid diagno- sis of infection in human immunodeficiency virus-infected patients who have unexplained fever an elevated serum levels of alkaline phosphatase or gamma-glutamyl transferase. Clin Infect Dis 1995; 26: 606-10.

13. Prego V, Glatt AE, Roy V, Thelmo W; Dincsoy H, Raufman JP. Comparative yield of blood culture for fungi and mycobacteria, liver biopsy, and bone marrow biopsy in the diagnosis of fever of undetermined origin in human immunodeficiency virus-infected patients. Arch Intern Med 1990; 150: 333-336.

14. Roger PM, Mondain V, Saint Paul MC, Carles M, Taillan B, Fuzibet JG, Michiels F, Dujardin P, Dellamonica P. Liver biopsy is not useful in the diagnosis of mycobacterial infections in patients who are infected with human immunodeficiency virus. Clin Infect Dis 1996; 23: 1302-1304.

15. Mayo J. Collazos J, Martinez E. Fever of unknown origin in the HIVinfected patient: new scenario for an old problem. Scand J Infect Dis 1997; 29: 327-336

16. Northfeld DW, Mayer A, Kaplan LD et al. The usefulness of diagnostic bone marrow examination in patients with human immunodeficiency virus (HIV) infection. J Acquir Immune Defic Syndr 1991; 4: 659-66.

17. Rodriguez JN, Dieguez JC, Moreno MV, Aguayo DM, Vega MD, Conde J, Prados D. Usefulness of bone marrow investigation in patients with advenced HIV infection. Rev Clin Esp 1996; 196: 213-216.

18. Engels E, Marks PW, Kazanjian P. Usefulness of bone marrow examination in the evaluation of unexplained fevers in patients infected with Human Immunodeficiency Virus. Clin Infect Dis 1995; 21:427-8.

19. Karcher DS, Frost AR. The bone marrow in human immunodeficiency virus (HIV)-related disease. Morphology an clinical correlation. Am J Clin Pathol 1991; 95: 63-71.

20. Raya Sánchez JM, Brito Barroso ML, Gómez Sirvent JL, Hernández Nieto L. Examen de médula ósea en pacientes con infección por virus de la unmunodeficiencia humana y giebre de origen desconocido. An Med Interna (Madrid) 1997; 14: 428-9.

21. Cabarcos Ortiz de Barrón A, Martínez Vázquez JM. Terapia combinada múltiple en el tratamiento de la infección por VIH: “¡Ha muerto la monoterapia, larga vida a la terapia combinada!”. An Med Interna (Madrid) 1998; 15: 121-124 